\title{
LIST OF PHOTOS
}

$\begin{array}{ll}\text { p. } 30 & \text { David Williams } \\ \text { p. } 60 & \text { Xander Tielens } \\ \text { p. } 102 & \text { Reception at the National Museum of Antiquities } \\ \text { p. } 128 & \text { Guillaume Pineau des Forêts } \\ \text { p. } 140 & \text { Tom Phillips } \\ \text { p. } 154 & \text { Alex Dalgarno, Mayo Greenberg, Stephen Lepp, Stefano Tiné } \\ \text { p. } 182 & \text { Conference dinner at "De Hoge Veluwe" } \\ \text { p. } 192 & \text { Drinks at "De Hoge Veluwe" } \\ \text { p. } 236 & \text { Maryvonne Gerin, Rolaine Young Owl } \\ \text { p. } 270 & \text { Ian Smith (back), Peter Bernath, Evelyne Roueff } \\ \text { p. } 340 & \text { Bill Langer, Willem Schutte } \\ \text { p. } 384 & \text { Poster session } \\ \text { p. } 396 & \text { Sculpture garden at the Kröller-Möller museum } \\ \text { p. } 420 & \text { Marco Spaans } \\ \text { p. } 456 & \text { Antonella Natta, Garrelt Mellema } \\ \text { p. } 476 & \text { Eric Herbst } \\ \text { p. } 504 & \text { Rafael Bachiller } \\ \text { p. } 514 & \text { Alex Dalgarno, Jonathan Rawlings, Stephen Lepp } \\ \text { p. } 534 & \text { Ewine van Dishoeck }\end{array}$

